saw cut out a $2 \mathrm{~mm}^{2}$ piece.

4) Prepare a mounting block by sawing off the pyramidal end of a blank resin block, previously polymerized in a BEEM capsule.

5) Glue the resin piece with the specimen to the prepared surface with cyanoacrylate glue. Orient as desired. Note: unpolymerized resin might work as well, but I have found that cyanoacrylate fastens the specimen in place quickly and surely, and without the sticky mess associated with resin.

6) Insert the block specimen-side up into a new BEEM capsule.

7) Cut the end off of a plastic soda-straw from your favorite fast-food restaurant and place the plastic cylinder around the specimen.

8) Reinforce the specimen by filling the cylinder with resin and polymerizing.

Dorothy Sorenson, Eastern Michigan University

\section{Preparation of Buffy Coat Leukocytes for Transmission Electron Microscopy}

The following procedure is an easy method to prepare buffy coat leukocytes for TEM. The cells are differentially centrifuged, resulting in layers enriched for each cell type. Granulocytes (eosinophils, neutrophils, and basophils) are found in the layer next to erythrocytes at the bottom of the buffy coat, monocytes and large lymphocytes are above the granulocytes, small lymphocytes are next, and platelets are at the top. Fixing the centrifuged cells without disturbing the layers results in a plug that can be processed easily while maintaining the cell layers.

Procedure:

1) Collect blood in standard EDTA tube.

2) Spin blood sample in clinical centrifuge to obtain a visible buffy coat (about $3500 \mathrm{rpm}$ for 20 minutes).

3) Carefully remove just the straw-colored plasma layer with pipette. A small amount of plasma may be left, if necessary, to avoid disturbing the buffy coat.

4) Gently add buffered glutaraldehyde by running it drop-wise down the sides of the tube, again without disturbing the buffy coat layer. $3 \%$ glutaraldehyde in $0.1 \mathrm{M}$ cacodylate or Sorensen's phosphate buffer, $\mathrm{pH} 7.2$ to 7.4 is suitable for primary fixation. Use this buffer in all buffer-only steps below.

5) After about 30 minutes, test the plug to see if it's fixed - it should feel rubbery with gentle prodding. If it is not firm, replace the fixative with fresh fixative and allow the buffy coat to fix for another 15 to 30 minutes.

6) Remove the fixed buffy coat plug from the tube. A small spatula or sharpened wooden applicator stick works well. This step can get a little messy, but the idea is to remove the plug as a coin-shaped disk with only a small number of adherent erythrocytes on the underside. Erythrocytes can be washed off with buffer if needed.

7) Place the plug with the erythrocyte-side down on dental wax and cut out a vertical slice. Take the slice and slice it again in the top-to-bottom direction into tissue-sized blocks ( 1 to $2 \mathrm{~mm}$ thick). Each block will contain all cell types layered as described above.

8) Place the blocks into a processing vial, and fix for an additional hour in glutaraldehyde.

9) Wash in $0.1 \mathrm{M}$ buffer, $2 \times 10$ minutes in each.

10) Post-fix in $1 \%$ osmium tetroxide in $0.1 \mathrm{M}$ buffer for 1 hour.

11) Rinse several times briefly in distilled water.

12) Dehydrate in ethanol: $50 \%, 70 \%$, and $95 \%, 15$ minutes in each.

13) Absolute ethanol: $2 \times 15$ minutes in each.

14) Propylene oxide: $2 \times 15$ minutes in each.

15) Propylene oxide: epoxy resin (1:1) for 1 hour.

16) $100 \%$ resin $-2 \times 8$ hours

17) Orient for embedding so that the largest face of the block will be sectioned (to get all the cell layers in the section).

18) Polymerize blocks as usual (at $60^{\circ} \mathrm{C}$ for 3 days or $90^{\circ} \mathrm{C}$ for 60 to 90 minutes).

Other dehydration and infiltration schedules will most likely work (e. g., acetone dehydration, different resins). Another variation is to remove the buffy coat before fixation and centrifuge it in an equal volume of fixative, thus eliminating platelets and erythrocytes while retaining the layering of leukocytes in embedded blocks (thanks to Ann Lehman at Trinity College, Hartford CT).

Reference: Anderson, D.R. 1965. A method for preparing peripheral leucocytes for electron microscopy. J. Ultrastructure Research 13:263-268.

Jane A. Fagerland, Abbott Laboratories

\section{Making Flexible Micrometer-Sized Needles}

We have used tungsten needles of about one micrometer with our high voltage electron microscope. What we were looking for was a rather rigid "needle point" on which to mount micrometer-sized objects. It was found that when we attempted to move these small specimens around and to get them to stick to the needles, the needles would bent very easily when they contacted the glass slides our specimens were prepared on. So tungsten would make good flexible needles.

We made them by electrolytically etching 5 mil. tungsten wires in $\mathrm{NaNO}_{3}$ solution:

1) Use a $30 \mathrm{~mL}$ beaker with $20 \mathrm{~mL}$ of roughly 0.5 molar $\mathrm{NaNO}_{3}$ with a two inch carbon evaporation electrode (1/8 inch diameter) connected to one side of a small 6.3 VAC (yes, $A C$ ) transformer and placed in the beaker.

2) 5 mil. tungsten wire was held in a small alligator test lead, with the other end connected to the other side of the transformer. The key part of the technique was to hold the alligator clip in hand and dip only the very tip of the tungsten wire into the solution (1/8 inch or less), as observed from below the interface. This had to be held steady for five minutes or so, then check the progress in the light microscope. Two or three iterations would usually suffice.

3) The wire was usually fused into a drawn glass tube for a handle.

Observing the results is the key. Also, once the tip of the wire is bent it would not return all the way to its being straight, which was at times frustrating.

Dave Barnard, New York State Department of Health, Wadsworth Center

A De-embedding Solution for $L X-112$ Resin

We at Ladd sell LX-112 and one procedure we know of that may work for de-embedding specimens in it is prepared as follows:

1) Prepare saturated solution of $\mathrm{KOH}$ in absolute ethanol. Let stand overnight.

2) Pour off supernatant fluid. This is the epoxy solvent.

3) Trim block of excess epoxy resin.

4) Let specimen soak in solvent until resin is removed. Time will vary depending on size of block.

5) Wash with several changes of absolute ethanol.

6) Prepare for SEM

Note: This solution may do damage to your specimen. I suggest trying this procedure on only one or two of your less critical samples.

Havat, M.A. (1989). Principles and Techniques of Electron Microscopy: Biological Applications. 3rd Edition. CRC Press, Inc. Boca Raton, Florida. 